\title{
Dihydroartemisinin inhibits HepG2.2.15 proliferation by inducing cellular senescence and autophagy
}

\author{
Jiang Zou, ${ }^{1,2,3, \#}$, Qiang Ma,2,3,\#, Ru Sun ${ }^{3,4}$, Jiajing Cai ${ }^{1,2,3}$, Hebin Liao ${ }^{2}$, Lei Xu ${ }^{2}$, Jingruo Xia ${ }^{1,2,3}$, Guangcheng Huang ${ }^{1,2,3}$, \\ Lihua Yao ${ }^{1,2,3}$, Yan Cai ${ }^{1,2,3}$, Xiaowu Zhong ${ }^{1,2,3, *} \mathcal{E}_{\text {Xiaolan Guo }}^{1,2,3, *}$ \\ ${ }^{1}$ Department of Clinical Laboratory, Affiliated Hospital of North Sichuan Medical College, ${ }^{2}$ Translational Medicine Research Center, North \\ Sichuan Medical College, ${ }^{3}$ Department of Laboratory Medicine, North Sichuan Medical College, ${ }^{4}$ Department of Blood Transfusion, \\ Affiliated Hospital of North Sichuan Medical College, Nanchong 637000, P.R.China
}

\begin{abstract}
Dihydroartemisinin (DHA) has been reported to possess anti-cancer activity against many cancers. However, the pharmacologic effect of DHA on HBV-positive hepatocellular carcinoma (HCC) remains unknown. Thus, the objective of the present study was to determine whether DHA could inhibit the proliferation of HepG2.2.15 cells and uncover the underlying mechanisms involved in the effect of DHA on HepG2.2.15 cells. We found that DHA effectively inhibited HepG2.2.15 HCC cell proliferation both in vivo and in vitro. DHA also reduced the migration and tumorigenicity capacity of HepG2.2.15 cells. Regarding the underlying mechanisms, results showed that DHA induced cellular senescence by up-regulating expression levels of proteins such as p-ATM, p-ATR, $\gamma-\mathrm{H}_{2} \mathrm{AX}, \mathrm{P} 53$, and P21 involved in DNA damage response. DHA also induced autophagy (green LC3 puncta gathered together and LC3II/LC3I ratio increased through AKT-mTOR pathway suppression). Results also revealed that DHA-induced autophagy was not linked to senescence or cell death. TPP1 (telomere shelterin) overexpression could not rescue DHA-induced anticancer activity (cell proliferation). Moreover, DHA down-regulated TPP1 expression. Gene knockdown of TPP1 caused similar phenotypes and mechanisms as DHA induced phenotypes and mechanisms in HepG2.2.15 cells. These results demonstrate that DHA might inhibit HepG2.2.15 cells proliferation through inducing cellular senescence and autophagy. [BMB Reports 2019; 52(8): 520-525]
\end{abstract}

\footnotetext{
*Corresponding authors. Xiaowu Zhong, Tel: +86-15882624081; Fax: +86-817-2282059; E-mail: zxw_strive@163.com; Xiaolan Guo, Tel: +86-817-2262302; Fax: +86-817-2282052; E-mail: alan 5200@hotmail.com

${ }^{\#}$ These authors contributed equally to this work.
}

https://doi.org/10.5483/BMBRep.2019.52.8.058

Received 24 February 2019, Revised 19 March 2019, Accepted 28 June 2019

Keywords: Autophagy, Cellular senescence, Dihydroartemisinin, DNA damage response, HCC

\section{INTRODUCTION}

Hepatocellular carcinoma (HCC) is the seventh most common malignancy worldwide and the second leading cause of cancer-related death (1). The incidence of HCC varies geographically. Most of its cases are found in developing countries. It has been reported that most cases of HCC are linked to chronic infections with hepatitis viruses, especially hepatitis B viruses (HBV) (2). Radiotherapy and chemotherapy are major treatments for HCC after surgical resection. Even with these treatments, median survival time of patients remains low. Recently, study of novel drugs or drug repurposing has become a major research focus in the field of HCC treatment.

Dihydroartemisinin (DHA), a semi-synthetic derivative of artemisinin, is a safe and WHO-recommended first-line antimalarial agent (3). In addition, DHA has no serious side effects on human body (4). DHA has been widely studied. Previous studies have shown that DHA has strong anticancer activities for different types of cancers. DHA could induce apoptosis of colorectal cancer cells (5) and suppress proliferation of pancreatic cancer cells via a microRNA-mRNA regulatory network (6). It can also inhibit proliferation of A549 lung cancer cells through AKT/GSK3ß/ cyclinD1 pathway (7) and induce apoptosis of BGC823 gastric cancer cells through activation of JNK1/2 and p38 MAPK signaling pathways (8). Some studies have reported that $\mathrm{DHA}$ could inhibit tumor growth by inducing ferroptosis and autophagy $(9,10)$. Although it is known that HBV is associated with liver cancer, very few studies have reported the effect of DHA treatment on HBV-positive $\mathrm{HCC}$ and the potential underlying mechanisms involved in such effect. Autophagy is an intracellular biological catabolic process of mammalian cells to maintain homeostasis. Recent studies have clearly demonstrated that dysfunction in autophagy could lead to the development of various kinds of diseases, including cancer (11). Thus, modulation of autophagy might have therapeutic value to cure cancer. Cellular senescence refers to irreversible cell cycle arrest that occurs in response to various forms of cellular stresses. It could be classified into oncogene-induced senescence (OIS) (12), stress induced premature senescence (SIPS) (13), and therapy induced senescence (TIS) (14). Many studies have suggested that cellular

ISSN: 1976-670X (electronic edition)

Copyright (C) 2019 by the The Korean Society for Biochemistry and Molecular Biology

(c) This is an open-access article distributed under the terms of the Creative Commons Attribution Non-Commercial License (http://creativecommons.org/licenses/by-nc/4.0) which permits unrestricted non-commercial use, distribution, and reproduction in any medium, provided the original work is properly cited. 
senescence is a key event contributing to anticancer response. It is clear that interaction between autophagy and senescence is required for DHA to alleviate liver fibrosis (15). However, whether cellular senescence could be induced after cancer cells are treated with DHA is currently unknown.

HepG2.2.15 is a liver cancer cell line capable of successfully expressing all viral markers of HBV. It is an important tool for studying HBV-associated HCC. To understand whether DHA might be a potential candidate chemotherapeutic reagent for $\mathrm{HBV}$-associated HCC, the objective of the present study was to determine the effect of DHA on proliferation of HepG2.2.15 cells in vitro and in vivo and uncover potential mechanisms involved in its effect on HBV-positive HCC.

\section{RESULTS}

DHA inhibits proliferation of HepG2.2.15 cells in vitro In order to investigate whether DHA might have anticancer effect on HCC, HepG2.2.15 cells were treated with a series concentration of DHA for 12, 24, 36, and 48 hours. Cellular phenotype was then observed under an optical microscope and cell proliferation was measured by crystal violet assay. Compared to cells treated with vehicle, some cells treated with DHA gradually became round and disconnected from dish after 24 hours of treatment. In addition, the number of dead cells was increased at 48 hours after treatment with DHA (Supplementary Fig. S1A). Results of crystal violet and CCK8 assay revealed that DHA could inhibit the proliferation of HepG2.2.15 cells in a timeand concentration-dependent manner $\left(\mathrm{IC}_{50}\right.$ value of $\mathrm{DHA}$ was approximately $100 \mu \mathrm{M}$, Supplementary Fig. S1B and S1C). These results suggested that $\mathrm{DHA}$ was an effective inhibition reagent against human HCC cells in vitro.

\section{DHA inhibits tumorigenicity of HepG2.2.15}

To further explore whether DHA treatment could affect the tumorigenicity of HepG2.2.15, soft agar colony formation assay and plate colony formation assay were employed to detect the tumorigenicity of HepG2.2.15 cells after treated with DHA. As
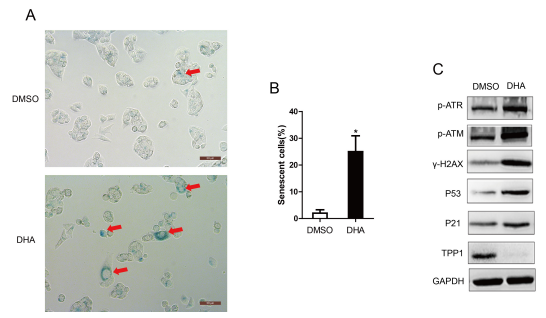

Fig. 1. DHA induces cellular senescence by triggering DNA damage response and downregulating telomere shelterin TPP1 expression at protein level. (A, B) SA- $\beta$-galactosidase assay (A) using cells treated with DHA $(100 \mu \mathrm{M})$ for $48 \mathrm{~h}$ (red arrows indicate senescent cells) and statistical chart (B). (C) Protein expression level of DDR signaling pathway and TPP1 after treatment with DHA $(100 \mu \mathrm{M})$ for $48 \mathrm{~h}$. shown in Supplementary Fig. S1D, the soft agar assay showed that the volume of colony spheres for DHA-treated cells was significant smaller than that for DMSO-treated cells. Plate colony formation assay also showed that DHA could decrease the growth of colony spheres (Supplementary Fig. S1E). These results showed that DHA could inhibit the tumorigenicity of HCC cells.

\section{Effect of DHA on HepG2.2.15 cells migration}

This study also investigated the migration ability of HepG2.2.15 cells after treatment with DHA. As illustrated in Supplementary Fig. S2A, wound healing assay showed that DHA remarkably decreased the migration ability of HepG2.2.15 cells. Similar results were obtained by performing transwell assay (Supplementary Fig. S2B and S2C). These data indicate that DHA might be an anti-metastasis reagent for treating hepatoma cells.

\section{DHA induces cellular senescence in HepG2.2.15 and its molecular mechanisms}

To further explore the underlying mechanisms involved in the effect of DHA on HepG2.2.15 cells, cellular senescence was determined by performing senescence associated $\beta$-galactosidase assay. Expression levels of proteins associated with senescence were measured by western blot. As demonstrated in Fig. 1A, exposure of HepG2.2.15 cells to DHA for 48 hours increased the number of senescent cells compared with control (exposure to DMSO). DNA damage response (DDR) pathway plays a key role in cellular senescence. Whether DDR is involved in HepG2.2.15 cellular senescence induced by DHA is unknown. As shown in Fig. 1C, compared with cells treated with vehicle, levels of p-ATM, p-ATR, $\gamma-\mathrm{H}_{2} \mathrm{AX}, \mathrm{P} 53$, and P21 were elevated while telomere shelterin TPP1 was significantly down-regulated in cells treated with DHA. These results demonstrate that DHA might induce HepG2.2.15 cellular senescence through DDR pathway.

\section{DHA induces autophagy in HepG2.2.15 cells by suppressing AKT/m-TOR pathway}

To determine whether DHA could induce autophagy in

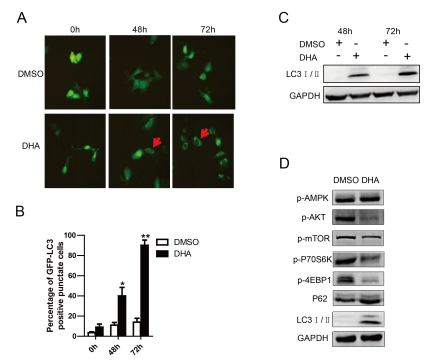

Fig. 2. DHA induces autophagy by inhibiting AKT-mTOR signaling pathway. (A, B) GFP-LC3 puncta (A) in DHA treated cells and statistical chart (B). (C) LC3 expression in HCC cells treated with DHA for 48 and 72 hours. (D) Expression levels of key proteins in AKT/m-TOR and autophagy signaling pathway. 
HepG2.2.15 cells, levels of autophagy marker LC3 were measured in cells treated with DHA. We transfected HepG2.2.15 cells with a GFP-LC3 plasmid using lipofectamine2000 reagent. At 24 hours after transfection, cells were treated with DHA or DMSO. At 48 hours post treatment, massive green LC3 puncta could be observed in cells treated with DHA compared to control (cells treated with DMSO) (Fig. 2A). Moreover, LC3, a marker of autophagy, was significant up-regulated in DHA treated group (Fig. 2C). A previous study has revealed that AKT-mTOR signaling is a key negative regulator of autophagy (16). In the present study, some key molecules in AKT-mTOR signaling were also detected by western blot. As shown in Fig. 2D, p-AKT, p-mTOR, p-p70S6K, and $\mathrm{p}-4 \mathrm{EBP} 1$ were down-regulated while autophagy associated protein LC3, p-AMPK, and p62 were up-regulated by DHA. These results suggest that DHA might induce autophagy in HepG2.2.15 cells though AKT-mTOR signaling suppression.

\section{DHA-induced autophagy is not linked to senescence or cell death}

To illustrate whether DHA-induced autophagy might be linked to senescence or cell death in HepG2.2.15 cells, we treated HepG2.2.15 cells with DHA after downregulating ATG5 by transfecting shRNA targeting human ATG5 gene followed by staining with crystal violet at the end of each treatment. There was no significant difference in proliferation or senescence of cells treated with DHA, regardless of whether ATG5 was downregulated or not (Supplementary Fig. S3C and S3G). These results demonstrate that DHA-induced autophagy is not linked to senescence or cell death.

Role of TPP1 in DHA-induced anticancer activity

To further clarify the role of TPP1 in DHA induced anticancer activity, we constructed lentivirus expressing vector harboring human TPP1 gene and transfected it into HepG2.2.15 cells. We

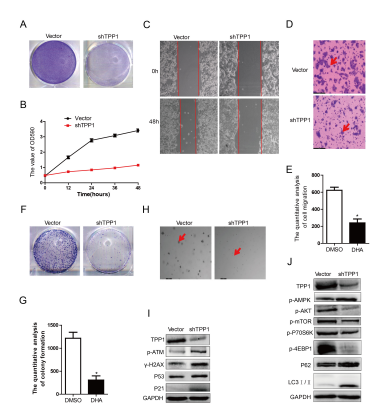

Fig. 3. Effects of TPP1 knockdown on HepG2.2.15 cells and its mechanisms. (A, B) Representative proliferation images (A) and growth curve (B) in TPP1 knockdown HepG2.2.15 cells. (C-E) Wound healing assay $(\mathrm{C})$, transwell assay $(\mathrm{D})$, and statistical chart $(\mathrm{E})$ showing migration capacity change in HepG2.2.15 cells treated with DHA or vehicle. $(\mathrm{F}-\mathrm{H})$ Plate colony formation assay $(\mathrm{F})$, statistical chart $(\mathrm{G})$, and soft agar assay $(\mathrm{H})$ showing altered tumorigenicity. $(\mathrm{I}, \mathrm{J})$ Modulation of key proteins in DDR pathway (I) and AKT-mTOR or autophagy signaling pathway (J) after TPP1 knockdown. then validated the expression of TPP1 by Western Blot. Subsequently, we treated HepG2.2.15 cells overexpressing TPP1 and control cells with DHA. Proliferation of HepG2.2.15 cells was then evaluated by crystal violet assay. We found that the difference in proliferation was not significant between cells overexpressing TPP1 and control cells after treatment with 100 $\mu \mathrm{M}$ DHA (Supplementary Fig. S4D). Moreover, SA- $\beta$-gal activity was analyzed after cells were treated with DHA for 24 hours. Results showed no obvious difference in the number of SA- $\beta$-gal positive cells between TPP1 overexpressing cells and control cells (Supplementary Fig. S5D). These results indicate that TPP1 overexpression does not affect DHA-induced anticancer activity.

Bioinformatics analysis of TPP1 in HCC from TCGA database From the above-mentioned results, we speculate that TPP1 might play a vital role in HepG2.2.15 cells exposure to DHA, although the expression of TPP1 in hepatoma tissue is unknown. Whether TPP1 is associated with clinical characteristic of liver cancer patients is also unclear. Thus, we employed an online tool named GEPIA (Gene Expression Profiling Interactive Analysis) to analyze expression difference of TPP1 at RNA level between hepatoma and adjacent tissues. As shown in Supplementary Fig. S6, the expression of TPP1 was elevated in HCC tissues (Supplementary Fig. S6A). High expression of TPP1 was associated with worse overall survival (Supplementary Fig. S6B). These results indicate that TPP1 might be a risk factor in HCC patients.

\section{The role of TPP1 in HepG2.2.15 cells}

To further elucidate the role of TPP1 in HepG2.2.15 cells, we utilized shRNA to mediate TPP1 and lentivirus expressing vector harboring human TPP1 gene to observe phenotype changes of HepG2.2.15 cells and explore the potential mechanisms. As shown in Fig. 3, the proliferation of HepG2.2.15 cells was arrested and their migration capacity was suppressed after TPP1 knockdown. Moreover, knockdown of TPP1 evidently inhibited the tumorigenicity of HepG2.2.15 cells in vitro. Further mechanisms study showed that genetic inhibition of TPP1 triggered ATM associated DDR and induced autophagy through AKT-mTOR signaling suppression. However, overexpression of TPP1 did not show obvious difference in cell growth or antitumor

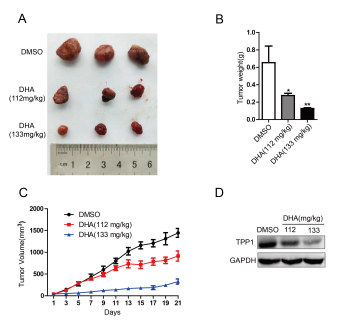

Fig. 4. DHA inhibits HepG2.2.15 proliferation in vivo. (A) Image of HCC from nude mice transplanted with HepG2.2.15 cells. (B, C) Tumor weight (B) and proliferation curve (C) of HCC xenograft from mice. (D) Expression of TPP1 from tumor tissue in xenograft mice. 
abilities. These results indicate that TPP1 might be positively associated with HCC.

\section{DHA exhibits antitumor effects in vivo}

To investigate whether DHA exhibited an effective antitumor activity in vivo, a xenograft model transplanted with HepG2.2.15 cells was treated with DMSO, $112 \mathrm{mg} / \mathrm{kg}$ of DHA, or $133 \mathrm{mg} / \mathrm{kg}$ of DHA. As illustrated in Fig. 4, DHA exhibited significant antitumor activity by suppressing tumor growth in a time- and dose- dependent manner. Meanwhile, DHA at $112 \mathrm{mg} / \mathrm{kg}$ or 133 $\mathrm{mg} / \mathrm{kg}$ significantly down-regulated telomere shelterin TPP1 expression. These results reveal that DHA could inhibit tumor growth though down-regulating TPP1 which might be an important molecule of DHA in HepG2.2.15.

\section{DISCUSSION}

To date, increasing research studies have focused on using DHA in cancer therapy, including therapy for HCC. A previous study has shown that DHA could inhibit proliferation and induce apoptosis of HepG2 cells by inducing endoplasmic reticulum stress (17). DHA could also increase apoptosis of HepG2 and Huh-7 cells via Bim-mediated pathway. In addition, it could inhibit Sp1 expression and increase apoptosis of SK-Hep-1 cells $(18,19)$. Moreover, DHA could suppress proliferation of HepG2 cells in vivo (20), demonstrating that DHA has the potential to be used as anti-cancer drug for liver tumors. Although anticancer effects of DHA on HCC have been reported, the molecular mechanisms involved in such effects remain unclear. In addition, different cancer cells have their unique genetic background. They might show different responses to the same drug through various molecular mechanisms. Thus, precision cancer therapy that can fine-tune treatment approach for cancer more precisely and match biology of the tumor is needed.

Chronic hepatitis B is the main cause of HCC in China. Whether HBV associated HCC is sensitive to DHA remains unknown. Thus, we utilized HepG2.2.15 to evaluate the anti-cancer effect of DHA on HBV-positive hepatocellular carcinoma in the present study. We found that DHA effectively inhibited proliferation, migration, and tumorigenicity of HepG2.2.15 cells in vitro. It also restrained tumor growth in vivo. These results demonstrate that DHA might be effective for HBV-positive HCC patients and other liver cancer types.

Therapy induced senescence (TIS) refers to senescence induced after cancer cells are exposed to chemotherapeutic drugs. Substantial evidence has shown that induction of senescence can inhibit cancer development (21). The present study showed that DHA increased cellular senescence of HepG2.2.15 cells which might be one of mechanisms involved in the effect of DHA on HCC. A previous study has shown that DDR pathway plays a key role in tumor cellular senescence process by up-regulating P53 and P21 to elicit growth arrest of MEFs and induce senescence (22). In the present study, we measured expression levels of proteins associated with DDR pathway. We found that DHA obviously elevated phosphorylation levels of ATM and ATR and increased expression levels of P53, P21, and $\gamma-\mathrm{H}_{2} \mathrm{AX}$ as key downstream molecules in DDR pathway. These results indicate that DHA might mediate HepG2.2.15 cellular senescence by inducing DDR.

Previous study have shown that DHA can induce autophagydependent cell death in various cancer cell lines in vitro $(9,23$, 24). Whether DHA could also induced autophagy in HepG2.2.15 needs to be clarified. In our study, green LC3 puncta significantly increased and protein expression level of LC3II/I was elevated after DHA treatment for 48 and 72 hours compared with vehicle control. AKT-mTOR signaling is known to be a key negative regulator of autophagy (16). Thus, we measured expression levels of p-AKT, p-mTOR, p-4EBP1, and p-P70 in DHA treated or untreated cells. Our results revealed that expression levels of p-AKT and p-mTOR were significantly down-regulated after treatment with DHA. Moreover, the level of $\mathrm{p}$-AMPK, a negative regulatory protein of m-TOR, was remarkably increased after treatment with DHA. Our current results indicate that DHA could induce autophagy in HepG2.2.15 cells via AKT-mTOR pathway suppression.

Our study showed that the difference in proliferation or senescence was not significant between cells treated with DHA and those treated with vehicle (DMSO) after autophagy was inhibited by downregulated ATG5. We then overexpressed TPP1 in HepG2.2.15 cells, treated cells with DHA or vehicle, and evaluated proliferation and senescence of cells. Our results showed that TPP1 overexpression did not affect DHA-induced anticancer activity. These results indicate that DHA induced autophagy, cellular senescence, and TPP1 downregulation might be independent of each other.

Our results also revealed that DHA down-regulated the expression of telomere shelterin TPP1 both in vivo and in vitro. TPP1 is a central component of shelterin that connects to POT1 through two essential POT1 C domains, the third OB fold, and Holliday junction resolvase-like domain (25). The essential function of TPP1 is to prevent ATR/ATM kinase dependent DNA damage response. Deletion of K170 in TPP1 is defective in stimulating telomerase enzymatic processivity which is a genetic foundation of Hoyeraal-Hreidarsson syndrome (26). Moreover, knockdown of TPP1 in MEFs can elicit p53-dependent growth arrest and ATM-dependent DDR at telomeres (22), revealing that TPP1 plays a vital role in normal cellular functional maintenance. Thus, targeting TPP1 might be a novel therapeutic approach for cancer. Bioinformatics analysis showed that the expression of TPP1 mRNA level was remarkably increased in HCC tissues compared to that in adjacent tissues. High expression of TPP1 was also found to be associated with worse overall survival to liver cancer patients. This reveals that TPP1 might be a risk factor of $\mathrm{HCC}$ and that down-regulating TPP1 in HCC might be an effective way to develop HCC therapy.

Besides pharmacologic inhibition, genetic knockdown by shRNA was employed to study the influence of TPP1 depletion on cellular phenotype and molecular change. We found that 
knockdown of TPP1 evidently inhibited cellular growth, migration, and tumorigenicity. Further studies showed that down-regulated TPP1 triggered ATM-associated DDR and induced autophagy by suppressing AKT-mTOR pathway, showing the same mechanisms as DHA. DHA might have exhibited anticancer effect by down-regulating telomere shelterin TPP1. However, further experiments are needed to uncover potentials of DHA in details in the near future.

In summary, the present study demonstrates that DHA exhibits effective anticancer activity against HBV-positive HCC. In addition, TPP1 might be a key molecule involved in the effect of DHA on HepG2.2.15 cells, although effects of DHA on telomere components need to be investigated in the near future.

\section{MATERIALS AND METHODS}

Detailed information is included in the Supplemental Material.

\section{ACKNOWLEDGEMENTS}

This study was supported by Scientific Research Fund of Sichuan Provincial Education Department (grant no. 16ZA0224), Scientific Research Fund of Sichuan Provincial Health and Family Planning Commission (grant no. 17PJ588), and Science and Technology Support Program of Nanchong (grant no. 16YFZJ0133, 18SXHZ0514, and 18SXHZ0579).

\section{CONFLICTS OF INTEREST}

The authors have no conflicting interests.

\section{REFERENCES}

1. Bray F, Ferlay J, Soerjomataram I, Siegel RL, Torre LA and Jemal A (2018) Global cancer statistics 2018: GLOBOCAN estimates of incidence and mortality worldwide for 36 cancers in 185 countries. CA Cancer J Clin 68, 394-424

2. Mokdad AA, Lopez AD, Shahraz S et al (2014) Liver cirrhosis mortality in 187 countries between 1980 and 2010: a systematic analysis. BMC Med 12, 145

3. Eastman RT and Fidock DA (2009) Artemisinin-based combination therapies: a vital tool in efforts to eliminate malaria. Nat Rev Microbiol 7, 864-874

4. Tong $\mathrm{Y}$, Liu $\mathrm{Y}$, Zheng $\mathrm{H}$ et al (2016) Artemisinin and its derivatives can significantly inhibit lung tumorigenesis and tumor metastasis through $\mathrm{Wnt} /$ beta-catenin signaling. Oncotarget 7, 31413-31428

5. Ontikatze T, Rudner J, Handrick R, Belka C and Jendrossek V (2014) Dihydroartemisinin is a hypoxia-active anti-cancer drug in colorectal carcinoma cells. Front Oncol 4, 116

6. Li Y, Wang Y, Kong R et al (2016) Dihydroartemisinin suppresses pancreatic cancer cells via a microRNA-mRNA regulatory network. Oncotarget 7, 62460-62473

7. Liao K, Li J and Wang Z (2014) Dihydroartemisinin inhibits cell proliferation via AKT/GSK3beta/cyclinD1 pathway and induces apoptosis in A549 lung cancer cells. Int J Clin Exp
Pathol 7, 8684-8691

8. Zhang S, Shi L, Ma H et al (2017) Dihydroartemisinin induces apoptosis in human gastric cancer cell line BGC-823 through activation of JNK1/2 and p38 MAPK signaling pathways. J Recept Signal Transduct Res 37, 174-180

9. Thongchot S, Vidoni C, Ferraresi A et al (2018) Dihydroartemisinin induces apoptosis and autophagy-dependent cell death in cholangiocarcinoma through a DAPK1-BECLIN1 pathway. Mol Carcinog 57, 1735-1750

10. Shi X, Wang L, Li X et al (2017) Dihydroartemisinin induces autophagy-dependent death in human tongue squamous cell carcinoma cells through DNA double-strand breakmediated oxidative stress. Oncotarget 8, 45981-45993

11. Amaravadi R, Kimmelman AC and White E (2016) Recent insights into the function of autophagy in cancer. Genes Dev 30, 1913-1930

12. Acosta JC and Gil J (2012) Senescence: a new weapon for cancer therapy. Trends Cell Biol 22, 211-219

13. Itahana K, Campisi J and Dimri GP (2004) Mechanisms of cellular senescence in human and mouse cells. Biogerontology 5, 1-10

14. Chang BD, Xuan Y, Broude EV et al (1999) Role of p53 and p21waf1/cip1 in senescence-like terminal proliferation arrest induced in human tumor cells by chemotherapeutic drugs. Oncogene 18, 4808-4818

15. Zhang Z, Yao Z, Zhao S et al (2017) Interaction between autophagy and senescence is required for dihydroartemisinin to alleviate liver fibrosis. Cell Death Dis 8, e2886

16. Wang SY, Yu QJ, Zhang RD and Liu B (2011) Core signaling pathways of survival/death in autophagy-related cancer networks. Int J Biochem Cell Biol 43, 1263-1266

17. Gao X, Luo Z, Xiang T, Wang K, Li J and Wang P (2011) Dihydroartemisinin induces endoplasmic reticulum stressmediated apoptosis in HepG2 human hepatoma cells. Tumori 97, 771-780

18. Im E, Yeo C, Lee HJ and Lee EO (2018) Dihydroartemisinin induced caspase-dependent apoptosis through inhibiting the specificity protein 1 pathway in hepatocellular carcinoma SK-Hep-1 cells. Life Sci 192, 286-292

19. Qin G, Zhao C, Zhang L et al (2015) Dihydroartemisinin induces apoptosis preferentially via a Bim-mediated intrinsic pathway in hepatocarcinoma cells. Apoptosis 20, 1072-1086

20. Zhang CZ, Zhang H, Yun J, Chen GG and Lai PB (2012) Dihydroartemisinin exhibits antitumor activity toward hepatocellular carcinoma in vitro and in vivo. Biochem Pharmacol 83, 1278-1289

21. Sun P, Yoshizuka N, New L et al (2007) PRAK is essential for ras-induced senescence and tumor suppression. Cell 128, 295-308

22. Guo X, Deng Y, Lin Y et al (2007) Dysfunctional telomeres activate an ATM-ATR-dependent DNA damage response to suppress tumorigenesis. EMBO J 26, 4709-4719

23. Liu X, Wu J, Fan M et al (2018) Novel dihydroartemisinin derivative DHA-37 induces autophagic cell death through upregulation of HMGB1 in A549 cells. Cell Death Dis 9, 1048

24. Qu C, Ma J, Liu X et al (2017) Dihydroartemisinin exerts anti-tumor activity by inducing mitochondrion and endoplasmic reticulum apoptosis and autophagic cell death 
in human glioblastoma cells. Mol Carcinog 11, 310

25. Chen C, Gu P and Wu J (2017) Structural insights into POT1-TPP1 interaction and POT1 C-terminal mutations in human cancer. Nat Commun 8, 14929
26. Kocak H, Ballew BJ, Bisht $K$ et al (2014) HoyeraalHreidarsson syndrome caused by a germline mutation in the TEL patch of the telomere protein TPP1. Genes Dev 28, 2090-2102 